\title{
Phase-field modeling of austenite grain size effect on martensitic transformation in stainless steels
}

\author{
Hemantha Kumar Yeddu ${ }^{\mathrm{a}, *}$ \\ ${ }^{a}$ School of Engineering, Newcastle University, Newcastle upon Tyne, NE1 7RU, United \\ Kingdom.
}

\begin{abstract}
A 2D elastoplastic phase-field model is developed to study the effect of prior austenite grain size on martensitic microstructure evolution in stainless steel. The effects of strain hardening and strengthening by grain size reduction (Hall-Petch effect) have been included in the model. The results show that martensite units form in different packets oriented in different crystallographic directions in simulated coarse grains, whereas uni-directional martensitic growth is observed in simulated fine grains. The number of packets and martensite block width increase with increasing grain size. With a decreasing grain size the martensitic transformation start temperature decreases, indicating strengthening of austenite. Once the transformation is initiated, at a given time, simulated fine grains give rise to higher volume fraction of martensite compared to simulated coarse grains. The von Mises equivalent stress and plastic strain are large in simulated fine grains compared to those in simulated coarse grains. The simulation results are in good agreement with experimental results.
\end{abstract}

\footnotetext{
*Corresponding Author. E-mail: hemanth.yeddu@ncl.ac.uk, hemu23@gmail.com Accepted for publication in Computational Materials Science, 20th July 2018.
} 
Keywords: phase-field model, martensitic transformation, microstructure, grain size, steels

\section{Introduction}

Steels are widely used as structural materials in a wide variety of industries, such as construction, automobile and aerospace. Due to the strong demand to reduce the weight of the structures with improved mechanical properties, such as strength and ductility, novel steels and steel processing methods are being developed. The new phases and the subsequent microstructure that form due to the phase transformations during the materials processing stages govern the mechanical properties. Hence the phase transformations need to be thoroughly understood in order to tailor the microstructure and mechanical properties. It is also essential to study the relationship between phase transformations and the different strengthening mechanisms, e.g. grain refinement, precipitation hardening.

Martensitic transformation (MT) is an important phase transformation that can occur in steels and many other engineering materials, such as Zr, Ti, shape memory alloys. MT in steels is a diffusionless solid state phase transformation of ductile austenite (FCC) into a high strength phase, known as martensite (BCC or BCT). Martensite can form athermally, i.e. during rapid quenching; isothermally by holding the steel close to the transformation temperature $\left(M_{s}\right)$; stress-assisted, i.e. by application of stress; strain-induced, i.e. by large deformation. MT can induce internal stresses and strains in the material and when these stresses exceed the yield limit, the material undergoes plastic deformation. Therefore, MT can induce dislocations in 
the material and can cause transformation induced plasticity (TRIP) effect, which can be utilized in enhancing the mechanical properties of the materials.

Martensite can form in the shape of laths or plates [1-3]. The two solid phases, austenite and martensite, are coherent and are governed by orientation relationships (OR). Based on the crystallography of the FCC-BCC phase transformation, Bain OR predicts the formation of 3 different martensite variants, Nishiyama-Wasserman OR predicts 12 variants and Kurdjumov-Sachs OR predicts 24 variants $[1,4,5]$. In case of lath martensite, different variants can form in groups and a hierarchic structure is observed in the experiments [1]. Martensite laths formed in an austenite grain can be grouped into several packets and within each packet there can be several blocks and sub-blocks. A group of laths that form along the same habit plane belong to the same packet. In a packet, two groups of laths with a small misorientation $\left(10^{\circ}\right)$ are collectively considered as a block, whereas each group of laths is considered as a sub-block $[1,6]$. Morito et al. have observed in their experiments on carbon steels that lath martensite forms in four packets, which contain 3 blocks each and within each block there are two sub-blocks. Since the packet and block boundaries are high angle boundaries, they can act as effective barriers for dislocation motion and hence can affect the strength and toughness of steels [6]. The yield strength increases with decreasing packet size in carbon steels [7].

On annealing, martensite can revert to austenite either in a martensitic (diffusionless) manner or through a diffusion-controlled mechanism. Reversed austenite formed in martensitic manner inherits dislocations from martensite and is beneficial in enhancing the mechanical properties [8]. Moreover, re- 
versed austenite inherits the lath-like structure from martensite, which leads to reduction in austenite grain size [9].

Grain refinement [8-12] and grain boundary strengthening [13] are reported to be effective strengthening mechanisms. Several studies have shown that martensitic transformation and reverse phase transformation of martensite to austenite can be utilized to design fine grain and ultra-fine grained steels $[9,10,12]$. Reduction of grain size has a considerable effect on martensite formation. Grain refinement can lead to reduction of $M_{s}$ temperature [14], increased retained austenite $[15,16]$ and dislocation density [17]. Grain refinement can lead to decrease in block width and packet size, although the change in lath width is small [6]. As the grain refinement leads to a decreased tendency to form martensite and can sometimes inhibit the transformation completely, it is difficult to study by experiments the effect of austenite grain size on $M_{s}$ temperature, morphology and mechanical properties [18]. Under extreme thermo-mechanical service conditions, martensitic transformation could occur in the stable fine and ultra-fine grained austenitic steels and hence this aspect needs to be accounted for during material design. Therefore, it is essential to understand the behavior of fine and ultra fine grains during martensitic transformation using theoretical approaches.

Due to its technical and scientific importance, MT has been thoroughly studied using mathematical models and experiments. The interaction between phase transformation, twinning and plasticity has been studied using phenomenological models $[19,20]$ and constitutive models based on continuum mechanics approaches [21-29]. The evolution of plastic strains due to martensitic transformation was also modeled [23, 25, 26, 28]. The phase- 
field approach [30-32] has been successfully applied to study martensitic transformation [33-46] as well as the reversion of martensite to austenite by shear mechanism [47]. The evolution of martensitic microstructure and dislocations using the phase-field approach has been studied [48, 49]. The phase-field approach was also applied to study the effect of austenite grain size on martensite formation in Fe-Ni alloy [40] and shape memory alloy [42]. However, the effect of grain size on martensitic transformation coupled with plasticity has not been studied using the phase-field method. In the present work, 2D phase-field model coupled with continuum plasticity is used to study the effect of grain size on martensite formation (FCC to BCC) in stainless steel [33]. As the focus of the present work is to study the evolution of martensite morphology at the microscopic level, continuum plasticity is considered instead of modeling the dislocations at nanoscale level as in Refs. [48, 49]. The effects of strain hardening and Hall-Petch effect (effect of grain size on yield strength) are included in the model. The results show the microstructure evolution along with the formation of different packets. The average block width as well as $M_{s}$ temperature for different grain sizes are also predicted. The present work also discusses the effect of grain refinement on the chemical driving forces for initiation and progression of the transformation.

\section{Phase-field model}

The phase-field equation governing the microstructure evolution is given by:

$$
\frac{\partial \eta_{p}}{\partial t}=-\sum_{q=1}^{q=v} L_{p q} \frac{\delta G}{\delta \eta_{q}}
$$


and

$$
\frac{\delta G}{\delta \eta_{p}}=\frac{\partial G_{v}}{\partial \eta_{p}}-\nabla \cdot\left(\boldsymbol{\beta} \cdot \nabla \eta_{p}\right)
$$

where $G$ is the Gibbs energy of the system, $\eta_{p}$ is the phase field variable that tracks the evolution of martensite, $v$ is the total number of martensite variants, $\beta$ is gradient coefficient and $L_{p q}$ is a matrix of kinetic parameters.

Martensite variants (laths), which form in 24 different crystallographic orientations, can be grouped into three basic variants known as Bain variants $[4,5]$. Bain variants are obtained when the cubic crystal is compressed along one of the three orthogonal axes, i.e. X, Y and Z, and is elongated along the other two axes. This gives rise to three compression possibilities, i.e. along $\mathrm{X}, \mathrm{Y}$ and Z, and hence three Bain variants can be obtained [5]. In the present two dimensional (2D) case, two phase-field variables $\left(\eta_{1}, \eta_{2}\right)$ that correspond to the two Bain variants with compression along X- and Y-axes, respectively are considered.

The Gibbs energy of a system undergoing athermal martensitic transformation can be expressed as:

$$
G=\int_{V}\left(G_{v}^{c h e m}+G_{v}^{g r a d}+G_{v}^{e l}\right) d V
$$

where $G_{v}^{\text {chem }}$ corresponds to the chemical part of the Gibbs energy density, $G_{v}^{g r a d}$ is the gradient energy term, $G_{v}^{e l}$ is the strain energy density.

$G_{v}^{c h e m}$ is expressed as a Landau-type polynomial [33, 34]:

$$
\begin{gathered}
G_{v}^{\text {chem }}\left(\eta_{1}, \eta_{2}\right)=\frac{1}{V_{m}}\left[\frac{1}{2} A\left(\eta_{1}^{2}+\eta_{2}^{2}\right)\right. \\
\left.-\frac{1}{3} B\left(\eta_{1}^{3}+\eta_{2}^{3}\right)+\frac{1}{4} C\left(\eta_{1}^{2}+\eta_{2}^{2}\right)^{2}\right]
\end{gathered}
$$

where $V_{m}$ is the molar volume and the coefficients $A, B, C$ are expressed in terms of Gibbs energy barrier $\left(\Delta G^{*}\right)$ and the driving force $\left(\Delta G_{m}\right)$ as: 
$A=32 \Delta G^{*}, B=\left(3 A-12 \Delta G_{m}\right), C=\left(2 A-12 \Delta G_{m}\right)$ and $\Delta G^{*}=\frac{V_{m} \beta}{2 \delta^{2}}[33]$. $\delta$ is the physical interface thickness, $V_{m}$ is molar volume and $\beta$ is gradient coefficient as explained below.

$G_{v}^{g r a d}$ is expressed as $[33,34]$ :

$$
G_{v}^{g r a d}=\frac{1}{2} \sum_{p=1}^{p=v} \beta_{i j}(p) \frac{\partial \eta_{p}}{\partial r_{i}} \frac{\partial \eta_{p}}{\partial r_{j}}
$$

where $\mathbf{r}(\mathbf{x}, \mathbf{y})$ is the position vector expressed in Cartesian coordinates. The term on the right hand side is same as the second term in Eq. (1b). $\beta_{i j}$ is the gradient coefficient matrix expressed in terms of the interfacial energy $(\gamma)$, molar volume and the Gibbs energy barrier. In this work isotropic interfacial properties are considered and it is also assumed that both austenitemartensite and martensite-martensite interfaces have same interfacial properties. Hence $\beta_{i j}(p)=\beta=\frac{9 \gamma^{2} V_{m}}{16 \Delta G^{*}}$.

$$
\begin{aligned}
& G_{v}^{e l} \text { can be expressed as }[33,34,37] \text { : } \\
& G_{v}^{e l}=\int_{\epsilon_{i j}^{0}(\mathbf{r})}^{\epsilon_{i j}(\mathbf{r})} \sigma_{i j}(\mathbf{r}) d \epsilon_{i j}(\mathbf{r})=\int_{\epsilon_{i j}^{0}(\mathbf{r})}^{\epsilon_{i j}(\mathbf{r})} c_{i j k l}\left(\epsilon_{k l}(\mathbf{r})-\epsilon_{k l}^{0}(\mathbf{r})-\epsilon_{k l}^{p l}(\mathbf{r})\right) d \epsilon_{i j}(\mathbf{r})
\end{aligned}
$$

where $\sigma_{i j}(\mathbf{r})$ is the stress, $c_{i j k l}$ is the tensor of elastic constants, $\epsilon_{i j}(\mathbf{r})$ is the total strain, $\epsilon_{k l}^{p l}(\mathbf{r})$ is the plastic strain. Small strain theory is assumed in the present work and therefore $\epsilon_{i j}(\mathbf{r})$ can be expressed as: $\epsilon_{i j}(\mathbf{r})=\frac{1}{2}\left(\frac{\partial u_{i}(\mathbf{r})}{\partial r_{j}}+\frac{\partial u_{j}(\mathbf{r})}{\partial r_{i}}\right)$, where $\mathbf{u}(\mathbf{r})$ is the local diplacement vector. Earlier works have considered finite strain theory $[23,28,35,48,49] . \epsilon_{i j}^{0}(\mathbf{r})$ is the stress-free transformation strain, given by:

$$
\epsilon_{i j}^{0}(\mathbf{r})=\sum_{p=1}^{p=2} \eta_{p}(\mathbf{r}) \epsilon_{i j}^{00}(p)
$$

where $\epsilon_{i j}^{00}(p)$ are the Bain strain tensors that govern the two phase-field variables $\left(\eta_{1}, \eta_{2}\right)$ and are given by: 


$$
\epsilon_{i j}^{00}(1)=\left[\begin{array}{cc}
\epsilon_{3} & 0 \\
0 & \epsilon_{1}
\end{array}\right], \epsilon_{i j}^{00}(2)=\left[\begin{array}{cc}
\epsilon_{1} & 0 \\
0 & \epsilon_{3}
\end{array}\right]
$$

where $\epsilon_{3}$ and $\epsilon_{1}$ are compressive and tensile transformation strains, respectively.

The material undergoes plastic deformation when the internal stress $\left(\sigma_{i j}(\mathbf{r})\right)$ exceeds the yield limit. In this work von Mises yield criterion is used. The evolution of plastic strain $\epsilon_{i j}^{p l}(\mathbf{r})$ is governed by [33, 37]:

$$
\frac{\partial \epsilon_{i j}^{p l}(\mathbf{r})}{\partial t}=-k_{i j k l} \frac{\delta G_{v}^{\text {shear }}}{\delta \epsilon_{k l}^{p l}(\mathbf{r})}
$$

where $G_{v}^{\text {shear }}$ is the shear energy density, $k_{i j k l}\left(=k c_{i j k l}^{-1}\right)$ is the plastic kinetic coefficient and $c_{i j k l}^{-1}$ is the compliance tensor. $k$ is a parameter, which controls the rate at which stresses are relaxed by means of plastic deformation and is called plastic relaxation rate. $k$ is predicted from the simulations such that the transformation occurs at the experimental $M_{s}$ temperature [50]. $G_{v}^{\text {shear }}$ is given by:

$$
G_{v}^{\text {shear }}=c_{i j k l}\left(\frac{1}{2} e_{i j}(\mathbf{r}) e_{k l}(\mathbf{r})+\frac{1}{2} e_{i j}^{0}(\mathbf{r}) e_{k l}^{0}(\mathbf{r})-e_{i j}(\mathbf{r}) e_{k l}^{0}(\mathbf{r})\right)
$$

where $e_{i j}(\mathbf{r})$ and $e_{i j}^{0}(\mathbf{r})$ are the deviatoric actual strain tensor and deviatoric stress-free transformation strain tensors given by Eq. (10a) and Eq. (10b), respectively.

$$
\begin{gathered}
e_{i j}(\mathbf{r})=\epsilon_{i j}(\mathbf{r})-\frac{1}{3} \epsilon_{k k}(\mathbf{r}) \delta_{i j} \\
e_{i j}^{0}(\mathbf{r})=\epsilon_{i j}^{0 t o t}(\mathbf{r})-\frac{1}{3} \epsilon_{k k}^{0 t o t}(\mathbf{r}) \delta_{i j}
\end{gathered}
$$

where $\epsilon_{i j}^{0 t o t}(\mathbf{r})$ is given by:

$$
\epsilon_{i j}^{0 t o t}(\mathbf{r})=\epsilon_{i j}^{0}(\mathbf{r})+\epsilon_{i j}^{p l}(\mathbf{r})
$$


Linear isotropic strain hardening is considered by using the following expression [51]:

$$
\sigma_{y}=\sigma_{y}^{0}+H \epsilon^{p l}(\mathbf{r})
$$

where $\sigma_{y}$ is yield stress of the material that depends on plastic strain, $\sigma_{y}^{0}$ is initial yield stress, $H$ is hardening modulus and $\epsilon^{p l}(\mathbf{r})$ is equivalent plastic strain.

Martensitic transformation in steels induces plastic deformation of prior austenite grain, whose yield stress depends on its size. Therefore, it is essential to include the effect of austenite grain size on the yield stress of austenite to study martensitic transformation in autenite grains of different sizes. HallPetch effect, i.e. increase in yield strength with decreasing austenite grain size, is considered by varying $\sigma_{y}^{0}$ of austenite in Eq. (12) as following [52-54]:

$$
\sigma_{y}^{0}=\sigma^{0}+\frac{k_{h}}{\sqrt{d}}
$$

where $\sigma_{y}^{0}$ is the initial yield stress of austenite, $\sigma^{0}$ is flow stress, $k_{h}$ is HallPetch slope and $d$ is austenite grain size in $\mu m$. The initial yield stress $\left(\sigma_{y}^{0}\right)$ of martensite does not vary with austenite grain size and only follows Eq. (12).

Finally the total strain is calculated by solving the mechanical equilibrium equation:

$$
c_{i j k l}\left(\frac{\partial \epsilon_{k l}}{\partial r_{j}}-\sum_{p=1}^{p=v} \epsilon_{k l}^{00}(p) \frac{\partial \eta_{p}(\mathbf{r})}{\partial r_{j}}-\frac{\partial \epsilon_{k l}^{p l}(\mathbf{r})}{\partial r_{j}}\right)=0
$$

In the present work, isotropic elasticity is considered by using the tensorial Hooke's relation between the stress and strain as:

$$
\sigma_{i j}(\mathbf{r})=\frac{E}{1+\nu}\left[\epsilon_{i j}^{e l}(\mathbf{r})+\frac{\nu}{1-2 \nu} \epsilon_{k k}^{e l}(\mathbf{r}) \delta_{i j}\right]
$$

where $E$ is Youngs modulus of elasticity and $\nu$ is Poisson ratio. 


\section{Simulation data}

The data, shown in Table-1, corresponding to stainless steels with a composition of Fe-17 wt \% Cr-7 wt \% Ni are acquired from different sources, such as CALPHAD, ab initio calculations and experiments [33, 39, 47, 52].

Simulations are performed on austenite single crystals of 1, 3, 5 and 10 $\mu m$ grain sizes by using FemLego software [59]. In the present work homogeneous isotropic elasticity is considered. A pre-existing spherical martensite $\left(\eta_{1}\right)$ embryo of $0.1 \mu \mathrm{m}$ is considered in the center of the grain. Since an individual grain in the bulk of a polycrystalline material is surrounded by other grains, Dirichlet (clamped) boundary conditions are considered in order to simulate the behavior of such a grain embedded in a polycrystalline material. Although Hall-Petch relationship is usually observed in polycrystalline materials, the choice of boundary conditions enables the yield stress to be modeled as grain size-dependent according to Hall-Petch relationship (Eq. (13)). The plastic relaxation rate $(k)$ is determined from the simulations for a given material and is independent of the grain size.

Different grid sizes with $100 \times 100,300 \times 300,500 \times 500$ and 1000 $\times 1000$ grid elements are considered to simulate grain sizes of $1,3,5$ and $10 \mu \mathrm{m}$, respectively. Hence the width of each grid element is $10 \mathrm{~nm}$. In the simulations three grid elements were considered as the interface width. Therefore, the actual interface width considered in the simulations is $30 \mathrm{~nm}$.

As the experimental data related to the mobility of the martensitic interface is ambiguous, the matrix of kinetic parameters $L_{p q}$ in Eq. (1a) that governs the mobility of the martensitic interface is considered to be an identity matrix. Due to the dependence of time $(t)$ on the ambiguous parameter 
Table 1: Simulation data.

Alloy composition: Fe-17 \% Cr-7 \% Ni, in weight percent

Grain sizes $=1,3,5$ and $10 \mu \mathrm{m}$

$M_{s}=263 \mathrm{~K}[55]$

Thermodynamic coefficients: $\mathrm{A}=1188 \mathrm{~J} / \mathrm{mol}, \mathrm{B}=3564 \mathrm{~J} / \mathrm{mol}$, $\mathrm{C}=2376 \mathrm{~J} / \mathrm{mol}[33]$

Gradient coefficient $(\beta)=0.1061 \times 10^{-10} \mathrm{~J} / \mathrm{m}[33]$

Driving force at $\mathrm{T}=263 \mathrm{~K}$ is: $-3600 \mathrm{~J} / \mathrm{mol}[56]$

Molar volume $\left(V_{m}\right)=7 \times 10^{-6} \mathrm{~m}^{3} / \mathrm{mol}$

Gibbs energy barrier $\left(\Delta G^{*}\right)=37.135 \mathrm{~J} / \mathrm{mol}$

Interfacial energy $(\gamma)=0.01 \mathrm{~J} / \mathrm{m}^{2}[34]$

Bain strains, $\epsilon_{1}=0.1316$ and $\epsilon_{3}=-0.1998$, are calculated from the lattice constants below.

Lattice constants: $a_{F C C}=3.5918 \AA, a_{B C C}=2.874 \AA[57]$

Youngs modulus of elasticity $(\mathrm{E})=200 \mathrm{GPa}$

Initial yield stress of austenite: $\sigma_{y}^{0}$ (austenite) $=684,456,385,315 \mathrm{MPa}$ for grains of size $1,3,5$ and $10 \mu m$, respectively.

$\sigma^{0}=144 \mathrm{MPa}, k_{h}=540 \mathrm{MPam}^{0.5}[52]$

Initial yield stress of martensite: $\sigma_{y}^{0}$ (martensite $)=800 \mathrm{MPa}$ [58]

Plastic relaxation rate $(\mathrm{k})=1.43 \mathrm{GPa}^{-1} \mathrm{~s}^{-1}$

$H=696,714,746,693 \mathrm{MPa}$ for grains of size 1, 3, 5 and $10 \mu \mathrm{m}$, respectively [52].

Interfacial kinetic coefficient $(\mathrm{L})=1 \mathrm{~m}^{3} \mathrm{~J}^{-1} \mathrm{~s}^{-1}$ 
$L_{p q}$, the time scale is expressed in terms of dimensionless time $t^{*}$.

\section{Results and discussion}

In the following discussion, prior austenite grains of size less than or equal to $2 \mu \mathrm{m}$ are termed as 'simulated fine grains' and grains of size greater than or equal to $3 \mu \mathrm{m}$ are termed as 'simulated coarse grains'. The evolution of microstructure at different time steps in grains of size $1 \mu \mathrm{m}$ and $10 \mu \mathrm{m}$ are shown in Figs. 1 and 2, respectively. The horizontal and vertical directions are considered to be along the $\mathrm{X}$ - and $\mathrm{Y}$-axes, respectively in all the simulated microstructures. The results show that martensite units grow in a single direction in simulated fine grains, whereas they grow in different directions in simulated coarse grains. In the simulated fine grains, two martensite units grow along a single crystallographic direction (Fig. 1). The simulated coarse grains give rise to martensite units growing in two different crystallographic directions (Fig. 2). The change in martensite morphology with austenite grain size is also observed in experiments $[18,60,61]$. The experiments by Takaki et al. showed that a coarse grained sample results in a microstructure with laths growing along different crystallographic directions whereas a finegrained material results in a microstructure with laths growing along a single crystallographic direction. A multi-variant transformation, which occurs in coarse grains can minimize the strain energy.

The local stresses play an important role in predicting the growth direction of martensite units by tilting the local energy balance between the chemical, gradient and strain energies. Fig. 3 shows that new martensite units nucleate and grow along a favorable direction, along which the min- 


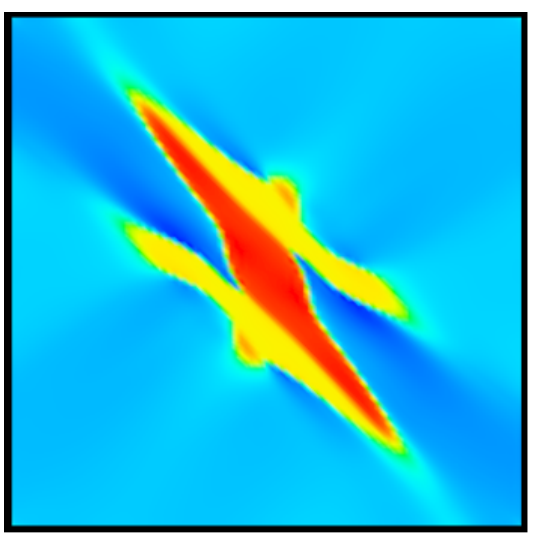

(a)

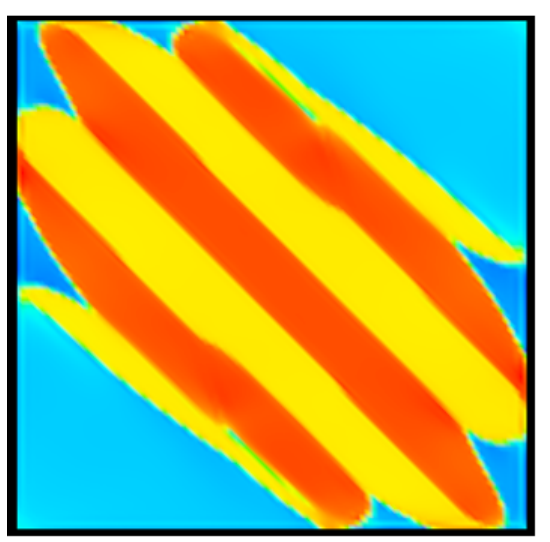

(c)

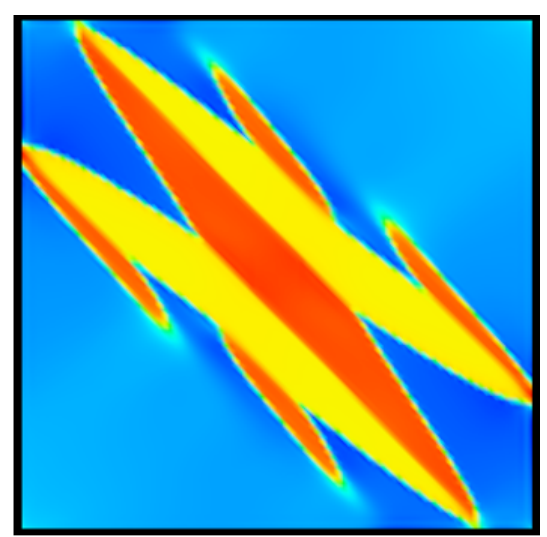

(b)

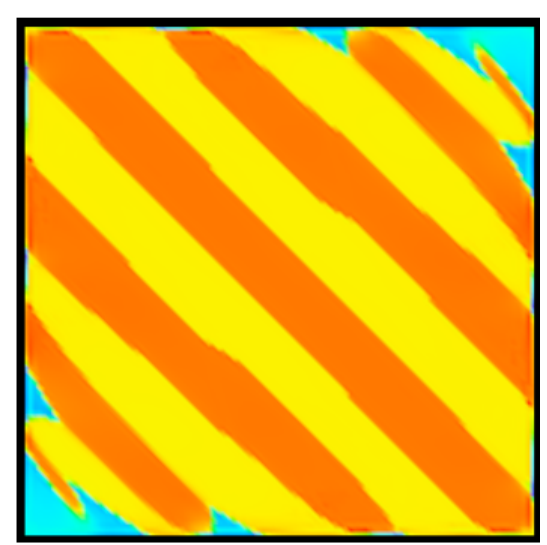

(d)

Figure 1: Simulated microstructures in a simulated fine grain (of 1 micron size) at dimensionless time $t^{*}=(\mathbf{a}) 2$ (b) 5 (c) 15 and (d) 30. Martensite variant-1 is shown in red/orange and variant-2 in yellow. Retained austenite is shown in blue. Horizontal direction is considered to be along the $\mathrm{X}$-axis and the vertical direction to be along the Y-axis. 


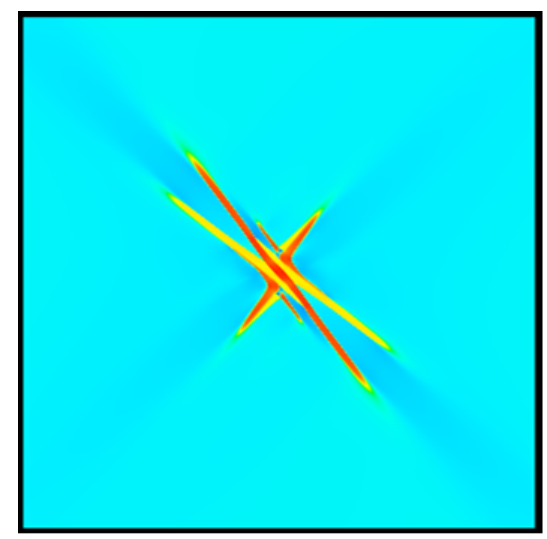

(a)

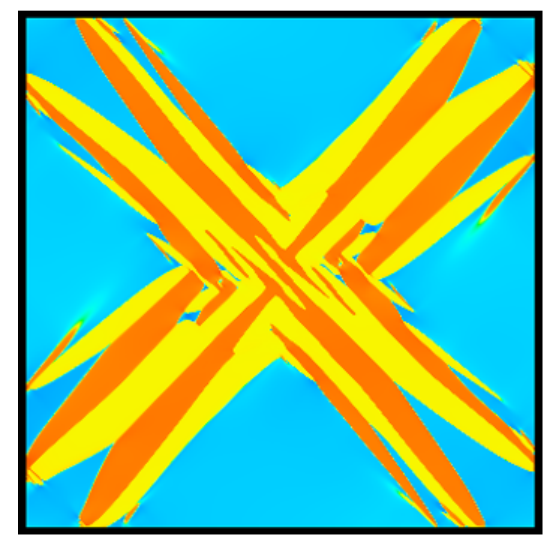

(c)

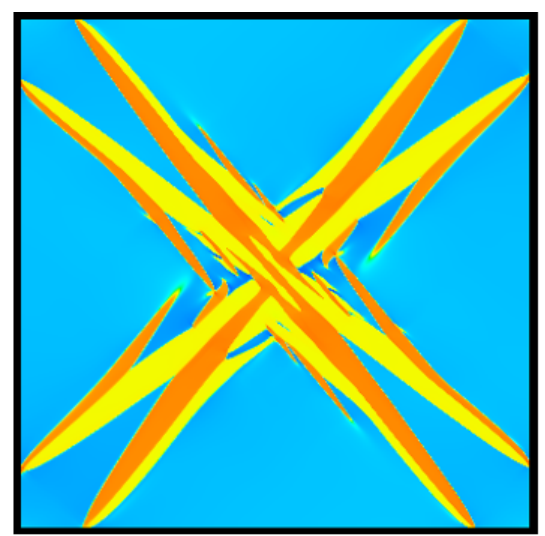

(b)

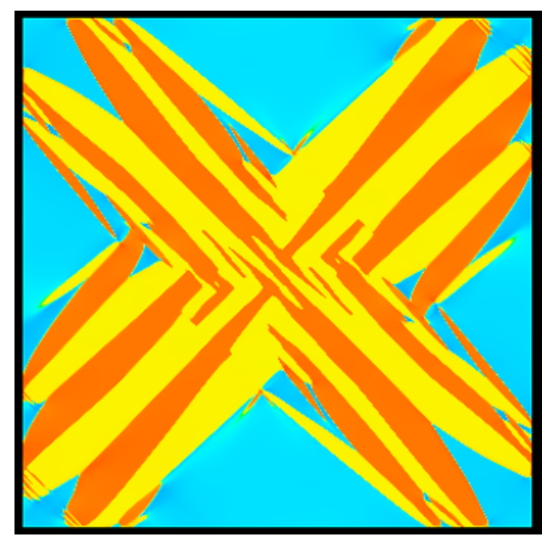

(d)

Figure 2: Simulated microstructures in a simulated coarse grain (of 10 micron size) at $t^{*}=$ (a) 5 (b) 15 (c) 25 and (d) 35. Martensite variant-1 is shown in red/orange and variant-2 in yellow. Retained austenite is shown in blue. 
imization of stresses is maximal. Fig. 3a and b shows that, in simulated fine grains, due to a large von Mises equivalent stress generated along the longitudinal direction of martensite units (arrow), the growth of martensite units continues in this direction (Fig. 3c) such that a maximum amount of stress generated ahead of the tip of marensite unit (Fig. 3b) is minimized. Fig. 3d and e shows that due to a large stress generated in the transverse direction of martensite units (arrow), new units grow along that direction in simulated coarse grains (Fig. 3f). Olson and Cohen have reported that the stress fields at the periphery of martensite units lead to autocatalytic nucleation of martensite laths [2]. Levitas et al. have also shown through their simulations that the internal stress plays a dominant role in the formation of self-accommodated martensite laths [3].

The microstructures obtained in different grains are shown in Fig. 4. The microstructures in Fig. 4b,c,d show that they contain different lath groups, which are separated by boundaries. This structure resembles the hierarchic lath martensitic structure found in steels $[1,6]$, as explained in Sec. 1. Although the simulated microstructures do not show the complete hierarchic structure, they show that there are four packets corresponding to the four different habit planes $\left(\{111\}^{\gamma}\right.$ planes) in an austenite grain (Fig. 4c,d). Fig. 5 shows that in a given $(111)^{\gamma}$ plane, according to Kurdjumov-Sachs OR, there are two martensite variants that are oriented parallel to each of the three directions of the $(111)^{\gamma}$ plane. This leads to the possibility of forming 6 variants for each of the four $\{111\}^{\gamma}$ planes and hence 24 different K-S variants can form in an austenite grain. Earlier theoretical and experimental studies $[1,4,62]$ reported that the two martensite variants shown in Fig. 5 belong to 


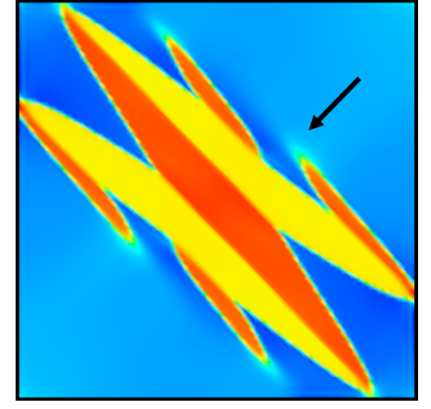

(a)

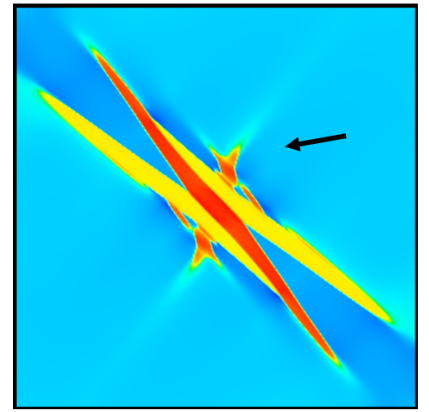

(d)

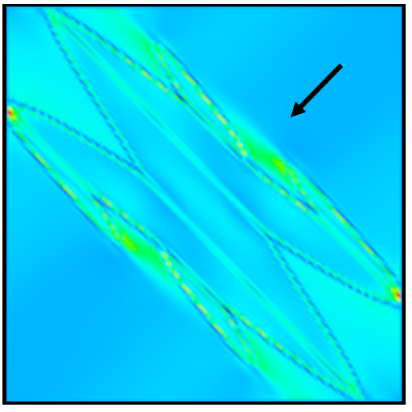

(b)

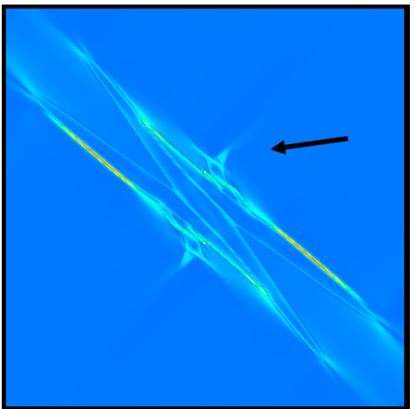

(e)

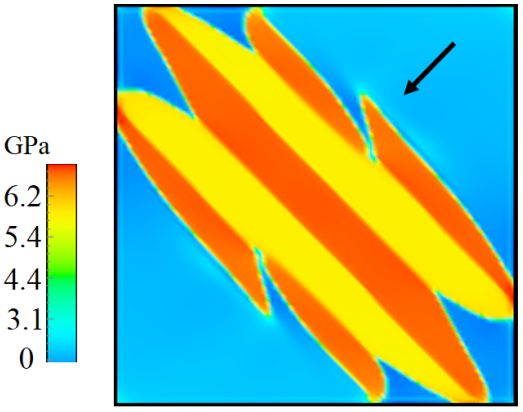

(c)

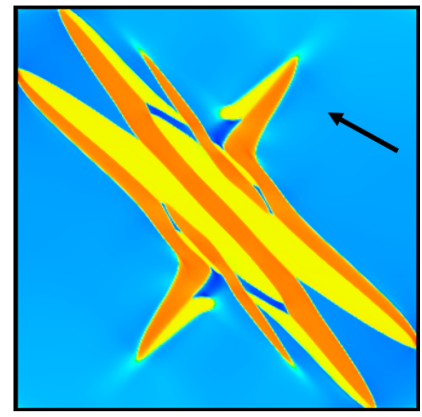

(f)

Figure 3: Austenite grain of $1 \mu \mathrm{m}$ size: (a) Microstructure and (b) corresponding von Mises equivalent stress at $t^{*}=5,(\mathbf{c})$ microstructure at $t^{*}=10$; Austenite grain of $3 \mu \mathrm{m}$ size: (d) Microstructure and (e) corresponding von Mises equivalent stress at $t^{*}=4.5,(\mathbf{f})$ microstructure at $t^{*}=10$. 
Table 2: Variation of the number of packets and block width with grain size.

\begin{tabular}{|c|c|c|}
\hline Grain size $(\mu m)$ & Number of packets & Block width $(\mu m)$ \\
\hline 1 & 1 & 0.082 \\
3 & 3 & 0.239 \\
5 & 4 & 0.281 \\
10 & 4 & 0.439 \\
\hline
\end{tabular}

two different blocks, i.e. B1 and B2. Guo et al. [4] and Morris [62] reported that the two blocks B1 and B2 are due to two different Bain strain tensors with compression along $\mathrm{X}$ - and $\mathrm{Y}$-axes, respectively. In the present work, since two Bain variants are considered in $2 \mathrm{D}$ with compression along $\mathrm{X}$ - and Y-axes (Eq. (7)) the two simulated martensite variants represent the two blocks B1 and B2 in Fig. 5.

Based on the above discussion, the width of each martensite variant is considered as the width of a block. This result is in agreement with Morito et al., who observed that in high carbon steels laths of single martensite variant can form as a block [1]. The width of a block in each packet is predicted by dividing the width of each packet by the number of martensite blocks in that packet (Fig. 6). The average of the four measurements corresponding to four packets is considered as the average width of martensite block in a grain. Table 2 shows that the average block width increases with increasing grain size, in good agreement with Ref. [6]. Fig. 4 and Table 2 also show that the number of packets in a grain increases with increasing grain size, in good agreement with Ref. [18]. 


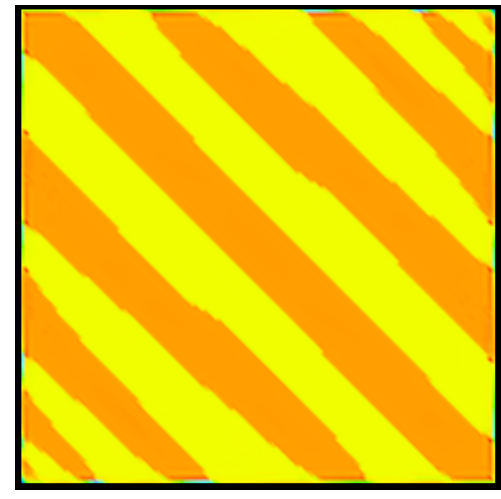

(a)

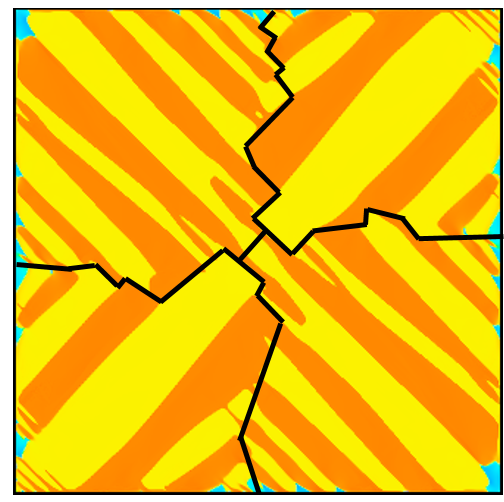

(c)

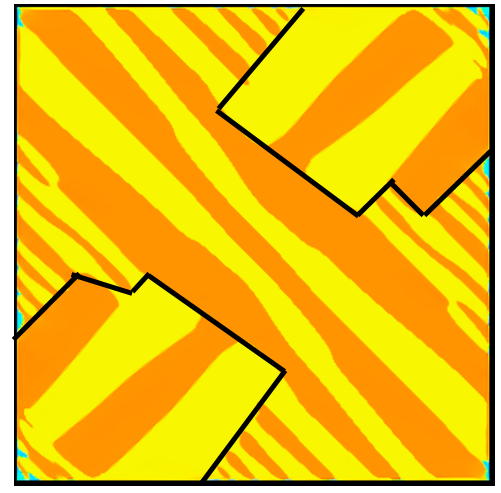

(b)

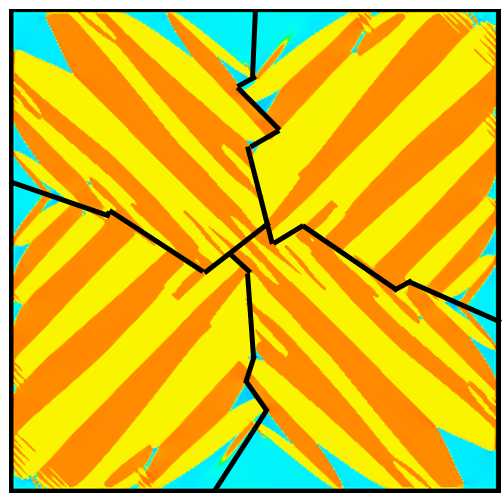

(d)

Figure 4: Simulated microstructures along with packet boundaries in grains of size (a) 1 $\mu m$ (b) $3 \mu m$ (c) $5 \mu m$ and (d) $10 \mu m$. Number of packets increase with increasing grain size. 


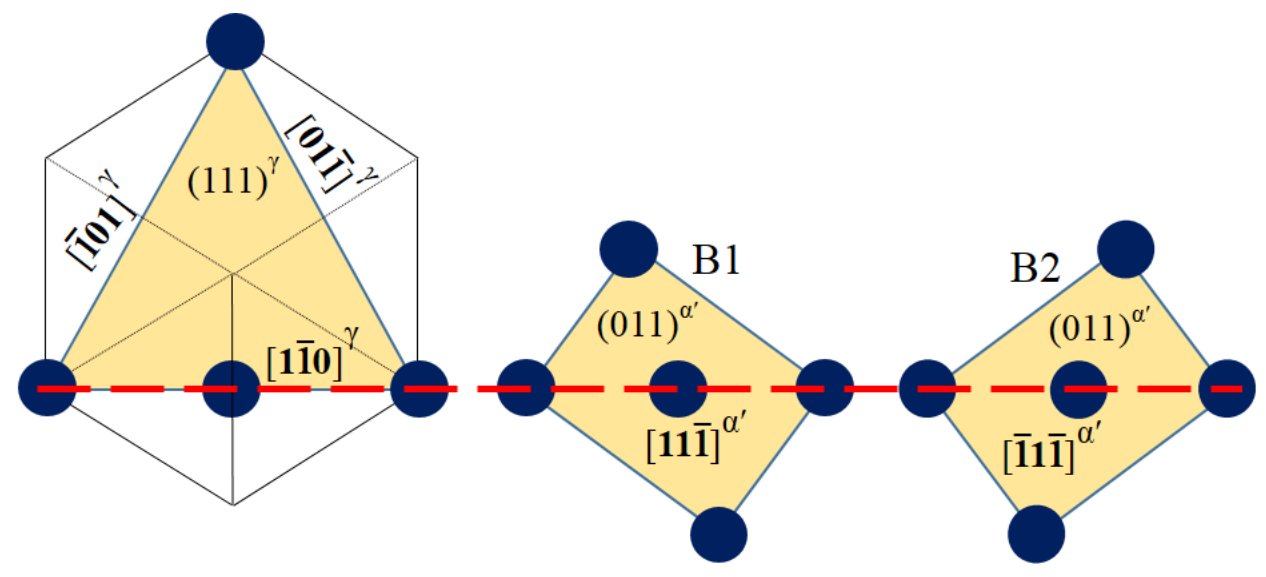

Figure 5: Kurdjumov-Sachs orientation relationship between austenite and martensite.

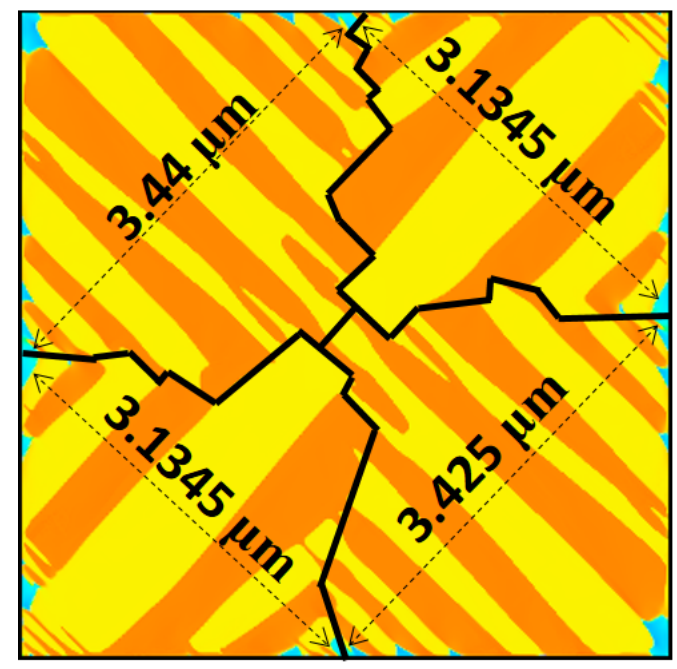

Figure 6: Procedure for measuring the width of a block in a 5 micron grain. 
Martensite start temperature $M_{s}$ decreases with decreasing grain size as shown in Fig. 7a, which is in good agreement with Refs. [14, 18, 63, 64]. This shows that austenite is more stable in simulated fine grains compared to that in simulated coarse grains. As $M_{s}$ decreases with decreasing grain size, the martensitic microstructure is refined as shown in Fig. 4 and in Table 1, also in good agreement with Ref. [6]. The evolution of the strain energy density in different grains is shown in Fig. $7 \mathrm{~b}$. In a simulated fine grain $(1 \mu m)$, the strain energy density is large during the initial stages of the transformation. This is because of the formation of martensite units along a single direction, which leads to poor accommodation of transformation stresses. In order to overcome such a large strain energy, a large thermodynamic driving force is needed. In the case of simulated coarse grains (3-10 $\mu \mathrm{m})$, as martensite grows along different directions the transformation stresses are minimized and hence simulated coarse grains do not require a large thermodynamic driving force to transform. Therefore $M_{s}$ temperature decreases with decreasing grain size, which is in good agreement with several experimental results [14, 18, 60, 63].

The large strain energy density during the intial stages of the transformation shows that it is difficult to initiate the transformation in simulated fine grains compared to simulated coarse grains (Fig. 7b) and hence a large chemical driving force is required for the initiation of transformation in simulated fine grains. This result is in good agreement with Takaki et al., who showed that the strain energy associated with the nucleation of thin plate martensite is very large in fine grains due to a single variant transformation compared to the coarse grains that undergo a multi-variant transformation [60]. During later stages of the transformation, the strain energy 


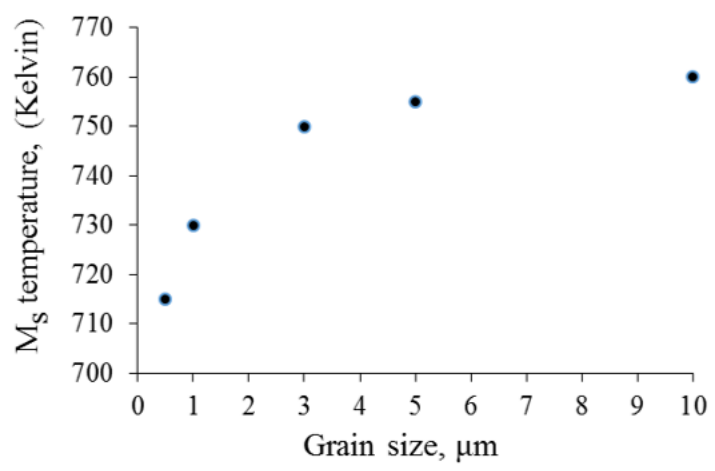

(a)

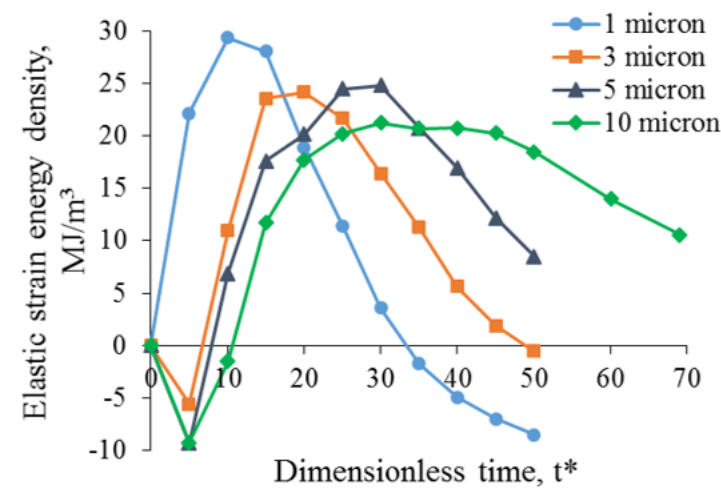

(b)

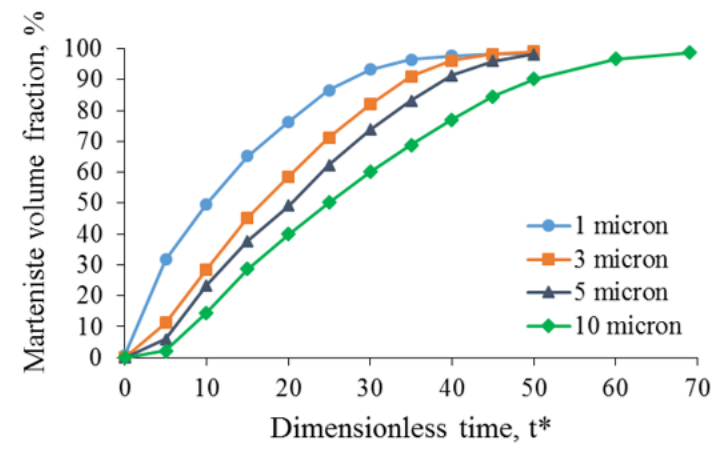

(c)

Figure 7: (a) Variation in Ms temperature with prior austenite grain size, (b) Evolution of strain energy density with $t^{*}$ in different grains, (c) evolution of martensite volume fraction with $t *$ in different grains. 
in simulated fine grains decreases whereas it increases in simulated coarse grains. The increased strain energy in simulated coarse grains implies a large chemical driving force is required for the progression of the transformation compared to that in simulated fine grains. The present results show that simulated fine grains require relatively large chemical driving force for initiation of transformation, whereas simulated coarse grains require relatively large chemical driving force for progression of the transformation. Previous studies $[50,65,66]$ have also shown that there exist two different driving forces for nucleation and growth of martensite. Therefore, grain refinement can decrease $M_{s}$ temperature, but once the transformation is initiated martensite volume fraction can increase rapidly in simulated fine grains compared to simulated coarse grains (Fig. 7c). Fig. 7c shows that the austenite grains of different size give rise to same amount of martensite at the end of quenching. However, at a given $t^{*}$ after the initiation of the transformation, simulated fine grains give rise to more volume fraction of martensite compared to simulated coarse grains. This is due to a relatively small chemical driving force required for the progression of the transformation in simulated fine grains, as explained above.

Simulated fine grains lead to larger internal stresses compared to simulated coarse grains as shown in Fig. 8a. The results are in good agreement with Matsuoka et al.'s experimental results on an Fe-16 wt.\%Cr-10 wt.\%Ni [61]. The large stresses generated in simulated fine grains are due to the absence of multi-directional martensitic growth. The stresses in simulated fine grains are minimized to a great extent through autocatalytic nucleation of numerous martensite variants and to a lesser extent through plastic accom- 
modation during the early stages of transformation. In the case of simulated coarse grains, multi-directional martensitic growth and plastic deformation are the dominant relaxation mechanisms during the early stages of transformation. Therefore, during early stages of transformation, the transformationinduced plastic strain for a given martensite volume fraction is slightly large in simulated coarse grain $(10 \mu \mathrm{m})$ compared to that in simulated fine grain (1 $\mu \mathrm{m})$ as shown in Fig. 8a and b. Levitas et al. [3] reported that plastic strains are large in materials with smaller yield stress. The present results corresponding to the early stages of the transformation are in good agreement with the predictions of Levitas et al. because the material with a smaller yield stress corresponds to a coarse grained material and the material with a larger yield stress corresponds to a fine grained material (Table 1).

During later stages of the transformation, plastic strain is large in simulated fine grains compared to simulated coarse grains. Due to the lack of formation of new martensite variants during the later stages of transformation as well as due to the absence of multi-directional martensitic growth in simulated fine grains, the stresses are mainly relaxed through plastic deformation. These results show that although the plastic strain is large in coarse-grained material during the early stages of the transformation, fine-grained material gives rise to a larger extent of plastic deformation towards the end of the transformation. It can be construed that plastic accommodation is slightly delayed in simulated fine grains compared to that in simulated coarse grains. Olson and Cohen have reported that the delay in plastic accommodation can also control the morphology of martensite, i.e. quick plastic accommodation 


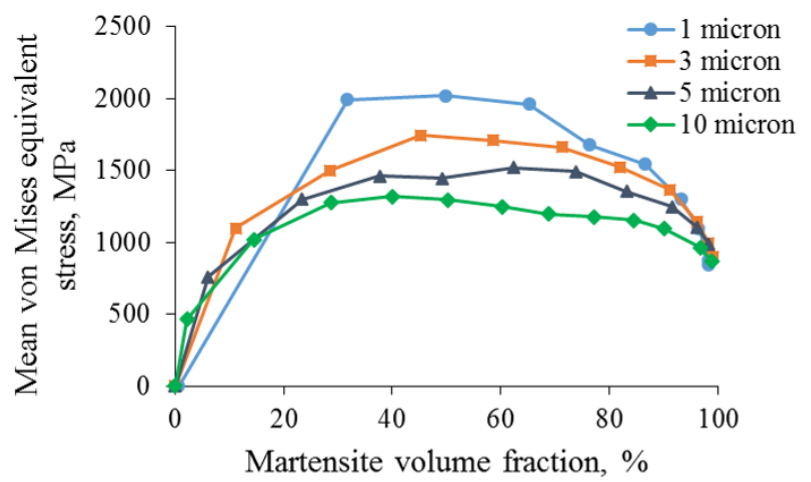

(a)

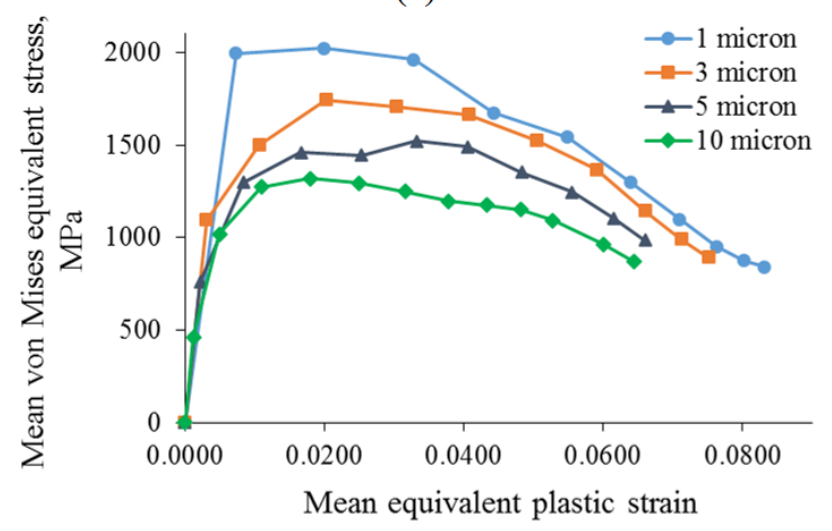

(b)

Figure 8: (a) Variation of mean von Mises equivalent stress with martensite volume fraction, (b) mean von Mises equivalent stress and mean equivalent plastic strain plots for different grains.

gives rise to lath martensite and delayed plastic accommodation gives rise to plate martensite [2].

\section{Conclusions}

The results show that martensite forms in a single crystallographic direction in simulated fine grains $(1 \mu \mathrm{m})$, whereas it forms in different crystallographic directions in simulated coarse grains $(\geq 3 \mu \mathrm{m})$. As multi-directional 
growth of martensite units can minimize the strain energy, new martensite units grow in the crystallographic direction along which the minimization of local stresses is maximal, as shown by the von Mises equivalent stress plots.

The simulated microstructures show that martensite can form along four crystallographic habit planes, thereby giving rise to a popular microstructural feature known as packets. Two martensite units form within each packet. On comparing the Bain orientation relationship (OR) considered in the present work with Kurdjumov-Sachs OR, it is concluded that the two martensite units belong to two different blocks. The results also show that the number of packets and the block width increase with increasing grain size.

The simulations predict that $M_{s}$ temperature decreases with decreasing grain size, indicating that austenite becomes more stable in fine grains. This is due to the decrease in the net driving force, which depends on the strain energy density that in turn depends on the growth pattern of martensite, i.e. uni-directional or multi-directional growth. The simulated fine grains require a large chemical driving force for initiation of transformation, whereas simulated coarse grains require large chemical driving force for the progression of transformation. Therefore, once the transformation is initiated, martensite volume fraction can increase rapidly in simulated fine grains compared to simulated coarse grains.

The uni-directional martensitic growth in simulated fine grains can generate large stresses. The transformation-induced plastic strain is relatively large in simulated coarse grains during early stages of transformation whereas it is large in simulated fine grains during later stages of transformation. These variations are attributed to the varying stress relaxation mechanisms, 
i.e. autocatalyic nucleation, plastic deformation, multi-directional martensitic growth or combinations of these mechanisms.

Based on the above results and discussion, it can be concluded that grain refinement leads to strengthening of austenite and hence initiation of martensitic transformation is difficult in fine grains compared to coarse grains. However, once the transformation is initiated, fine grains provide much more favorable conditions for progression of the transformation compared to coarse grains.

\section{Acknowledgements}

This work made use of the facilities of N8 HPC Centre of Excellence, provided and funded by the N8 consortium and EPSRC (Grant No. EP/K000225/1). The Centre is co-ordinated by the Universities of Leeds and Manchester.

\section{Data Availability}

The raw data required to reproduce these findings is presented in Sec. 3 .

\section{References}

[1] S. Morito, H. Tanaka, R. Konishi, T. Furuhara, T. Maki, The morphology and crystallography of lath martensite in Fe-C alloys, Acta Mater. 51 (2003) 1789-1799.

[2] G.B. Olson, M. Cohen, Dislocation theory of martensitic transformations. In: Nabarro, F.R.N.(Ed.), Dislocations in Solids, vol.7. NorthHolland, Amsterdam, 1986, pp. 297-407. 
[3] V.I. Levitas, A.V. Idesman, G.B. Olson, E. Stein, Numerical modeling of martensite growth in elastoplastic material, Philos. Mag. A82 (2002) 429-462.

[4] Z.Guo, C.S.Lee, J.W.Morris Jr., On coherent transformations in steel, Acta Mater. 52 (2004) 5511-5518.

[5] H.K. Yeddu, PhD thesis, Martensitic transformations in steels - A 3D phase-field study, KTH Royal Institute of Technology, Sweden, 2012.

[6] S. Morito, H. Saito, T. Ogawa, T. Furuhara, T. Maki, Effect of prior austenite grain size refinement by thermal cycling on the microstructural features of as-quenched lath martensite, ISIJ Int. 45 (2005) 91-94.

[7] T. Swarr and G. Krauss, The effect of structure on the deformation of as-quenched and tempered martensite in an Fe-0.2 pct $\mathrm{C}$ alloy, Metall. Trans. A 7 A (1976) 41-48.

[8] K. Tomimura, S. Takaki, Y. Tokunaga, Reversion mechanism from deformation induced martensite to austenite in metastable austenitic stainless steels. ISIJ Int. 31 (1991) 1431-1437.

[9] R.D.K. Misra, Z. Zhang, P.K.C. Venkatasurya, M.C. Somani, L.P. Karjalainen, Martensite shear phase reversion-induced nanograined/ultrafine-grained Fe-16Cr-10Ni alloy: The effect of interstitial alloying elements and degree of austenite stability on phase reversion, Mater. Sci. Eng. A 527 (2010) 7779-7792.

[10] F. Forouzan, A. Najafizadeh, A. Kermanpur, A. Hedayati, R. Surkialiabad, Production of nano/submicron grained AISI 304L stainless steel 
through the martensite reversion process, Mater. Sci. Eng. A 527 (2010) 7334-7339.

[11] H. Shirazi, G. Miyamoto, S.H. Nedjad, H.G. Nanesa, M.N. Ahmadabadi, T. Furuhara, Microstructural evaluation of austenite reversion during intercritical annealing of Fe-Ni-Mn martensitic steel, J. Alloys Compd. 577 (2013) S572-S577.

[12] A.D. Schino, M. Barteri, J.M. Kenny, Development of ultra fine grain structure by martensitic reversion in stainless steel, J. Mat. Sci. Lett. 21 (2002) 751-753.

[13] D. Raabe, S. Sandlöbes, J. Millan, D. Ponge, H. Assadi, M. Herbig, P.P. Choi, Segregation engineering enables nanoscale martensite to austenite phase transformation at grain boundaries: a pathway to ductile martensite, Acta Mater. 61 (2013) 6132-6152

[14] S.M.C. Van Bohemen and J. Sietsma, Kinetics of martensite formation in plain carbon steels: critical assessment of possible influence of austenite grain boundaries and autocatalysis, Mater. Sci. Tech. 30 (2014) 1024-1033.

[15] W. Hui, Microstructure refining and strengthening of martensitic steel, in: Yuqing Weng (Ed.), Ultra-Fine Grained Steels, Springer Berlin Heidelberg, 2009, pp. 300-349.

[16] J. Hidalgo and M.J. Santofimia, Effect of prior austenite grain size refinement by thermal cycling on the microstructural features of as-quenched lath martensite, Met. Mater. Trans. A 47 (2016) 5288-5301. 
[17] S.C. Kennett, G. Krauss, K.O. Findley, Prior austenite grain size and tempering effects on the dislocation density of low-C Nb-Ti microalloyed lath martensite, Scripta Mater. 107 (2015) 123-126.

[18] T. Hanamura, S. Torizuka, S. Tamura, S. Enokida, H. Takechi, Effect of Austenite Grain Size on Transformation Behavior, Microstructure and Mechanical Properties of 0.1C5Mn Martensitic Steel, ISIJ Int. 53 (2013) 2218-2225.

[19] J.R.C. Guimaraes, P.R. Rios, The mechanical-induced martensite transformation in FeNiC alloys, Acta Mater. 84 (2015) 436-442.

[20] M.X. Huang, O. Bouaziz, D. Barbier, S. Allain, Modelling the effect of carbon on deformation behavior of twinning induced plasticity steels, J. Mater. Sci. 46 (2011) 7410-7414.

[21] H. Hallberg, P. Håkansson, M. Ristinmaa, A constitutive model for the formation of martensite in austenitic steels under large strain plasticity, Int. J. Plast. 23 (2007) 1213-1239.

[22] A.L. Roitburd, D.E. Temkin, Plastic deformation and thermodynamic hysteresis at phase transformations in solids, Sov. Phys. Solid State 28 (1986) 432-436.

[23] I.M. Kaganova, A.L. Roitburd, Defects heredity and the phase transformation development in solids, Sov. Phys. Solid State 29 (1987) 800-803.

[24] I.M. Kaganova, A.L. Roitburd, Effect of plastic deformation on the equilibrium shape of a new phase inclusion and thermodynamic hysteresis, Sov. Phys. Solid State 31 (1989) 545-550. 
[25] V.I. Levitas, Phase transitions in elastoplastic materials: continuum thermomechanical theory and examples of control- Part I, J. Mech. Phys. Solids 45 (1997) 923-947.

[26] V.I. Levitas, Phase transitions in elastoplastic materials: continuum thermomechanical theory and examples of control- Part II, J. Mech. Phys. Solids 45 (1997) 1203-1222.

[27] F.D. Fischer, G. Reisner, A criterion for the martensitic transformation of a microregion in an elastic-plastic material, Acta Mater. 46 (1998) 2095-2102.

[28] V.I. Levitas, Thermomechanical theory of martensitic phase transformations in inelastic materials, Int. J. Solids Struct. 35 (1998) 889-940.

[29] F.D. Fischer, G. Reisner, E. Werner, K. Tanaka, G. Cailletaud, T. Antretter, A new view on transformation induced plasticity (TRIP), Int. J. Plast. 16 (2000) 723-748.

[30] N. Moelans, B. Blanpain, P. Wollants, An introduction to phase-field modeling of microstructure evolution, CALPHAD 32 (2008) 268-294.

[31] L.Q. Chen, Phase-field models for microstructure evolution, Annu. Rev. Mater. Res. 32 (2002) 113-140.

[32] S. Minamoto, S. Nomoto, A. Hamaya, T. Horiuchi, S. Miura, Microstructure simulation for solidification of magnesium-zinc-yttrium alloy by multiphase-field method coupled with CALPHAD database, ISIJ Int. 50 (2010) 1914-1919. 
[33] H.K. Yeddu, A. Malik, J. Ågren, G. Amberg, A. Borgenstam, Threedimensional phase-field modeling of martensitic microstructure evolution in steels, Acta Mater. 60 (2012) 1538-1547.

[34] A. Artemev, Y. Jin, A.G. Khachaturyan, Three-dimensional phase field model of proper martensitic transformation, Acta Mater. 49 (2001) 11651177.

[35] V.I. Levitas, A.V. Idesman, G.B. Olson, E. Stein, Numerical modeling of martensite growth in elastoplastic material, Philos. Mag. A 82 (2002) 429-462.

[36] H.K. Yeddu, A. Borgenstam, J. Ågren, Effect of martensite embryo potency on the martensitic transformations in steels - A 3D phase-field study, J. Alloys. Compd. 577S (2013) S141-S146.

[37] A. Yamanaka, T. Takaki, Y. Tomita, Elastoplastic phasefield simulation of self- and plastic accommodations in cubic tetragonal martensitic transformation, Mater. Sci. Eng. A 491 (2008) 378-384.

[38] H.K. Yeddu, A. Borgenstam, J. Ågren, Stress-assisted martensitic transformations in steels: a 3-D phase-field study, Acta Mater. 61 (2013) 2595-2606.

[39] H.K. Yeddu, V.I. Razumovskiy, A. Borgenstam, P.A. Korzhavyi, A.V. Ruban, J. Ågren, Multi-length scale modeling of martensitic transformations in stainless steels, Acta Mater. 60 (2012) 6508-6517.

[40] S. Cui, Y. Cui, J. Wan, Y. Rong, J. Zhang, Grain size dependence of 
the martensite morphology - a phase-field study, Comp. Mat. Sci. 121 (2016) 131-142.

[41] Y. Tsukada, Y. Kojima, T. KOYAMA, Y. MURATA, Phase-field Simulation of Habit Plane Formation during Martensitic Transformation in Low-carbon Steels, ISIJ Int. 55 (2015) 24552462.

[42] R. Ahluwalia, S.S. Quek, D.T. Wu, Simulation of grain size effects in nanocrystalline shape memory alloys, J. App. Phy. 117 (2015) 244305.

[43] J. Kundin, H. Emmerich, J. Zimmer, Three-dimensional model of martensitic transformations with elasto-plastic effects, Phil. Mag. 90 (2010) 14951510.

[44] T.W. Heo, L.Q. Chen, Phase-field modeling of displacive phase transformations in elastically anisotropic and inhomogeneous polycrystals, Acta Mater. 76 (2014) 68-81.

[45] H.K. Yeddu, H. Zong, T. Lookman, Alphaomega and omegaalpha phase transformations in zirconium under hydrostatic pressure: A 3D mesoscale study, Acta Mater. 102 (2016) 97-107.

[46] H.K. Yeddu, T. Lookman, Phase-field modeling of the beta to omega phase transformation in ZrNb alloys, Mater. Sci. Eng. A 634 (2015) $46-54$.

[47] H.K. Yeddu, T. Lookman, A. Saxena, Reverse phase transformation of martensite to austenite in stainless steels: a 3D phase-field study, J. Mater. Sci. 49 (2014) 3642-3651. 
[48] V.I. Levitas, M. Javanbakht, Interaction between phase transformations and dislocations at the nanoscale Part 1 General phase field approach, J. Mech. Phys. Solids 82 (2015) 287-319.

[49] M. Javanbakht, V.I. Levitas, Interaction between phase transformations and dislocations at the nanoscale Part 2 Phase field simulation examples, J. Mech. Phys. Solids 82 (2015) 164-185.

[50] H.K. Yeddu, A. Borgenstam, P. Hedstrom, J. Ågren, A phase-field study of the physical concepts of martensitic transformations in steels, Mater. Sci. Eng. A 538 (2012) 173-181.

[51] E.A. De souza Neto, D. Peric, D.R.J. Owen, Computational methods for plasticity - Theory and applications, John Wiley and Sons Ltd., West Sussex (UK), 2008.

[52] A.D. Schino, I. Salvatori, J.M. Kenny, Effects of martensite formation and austenite reversion on grain refining of AISI 304 stainless steel, J. Mat. Sci. 37 (2002) 4561-4565.

[53] L.P. Karjalainen, T. Taulavuori, M. Sellman, A. Kyrolainen, Some strengthening methods for austenitic stainless steels, Steel Res. Int. 79 (2008) 404-412.

[54] S. Rajasekhara, P.J.Ferreira, L.P. Karjalainen, A.Kyrolainen, Hall-Petch behavior in ultra-fine-grained AISI 301LN stainless steel, Met. Mater. Trans. A 38A (2007) 1202-1210.

[55] B.P.J. Sandvik, H.O. Martikainen, V.K. Lindroos, The crystallography 
and microstructure of lath martensite formed in type 301 stainless steel, Scripta Metall 18 (1984) 81-86.

[56] J.O. Andersson, T. Helander, L. Höglund, P.F. Shi, B. Sundman, THERMO-CALC \& DICTRA computational tools for materials science, CALPHAD 26 (2002) 273-312.

[57] P. Sahu, Bainite and stress-induced martensite in an AISI type 300 steel: an X-ray diffraction study of the microstructure by the Rietveld method, J Appl Crystallogr 38 (2005) 112-120.

[58] G. Krauss. Martensite in steel: strength and structure, Mater Sci Eng A 273-275 (1999) 40-57.

[59] G. Amberg, R. Tönhardt, C. Winkler, Finite element simulations using symbolic computing, Math. and Comp. in Sim. 49 (1999) 257-274.

[60] S. Takaki, K. Fukunaga, J. Syarif, T. Tsuchiyama, Effect of grain refinement on thermal stability of metastable austenitic steel, Mater. Trans. 45 (2004) 2245-2251.

[61] Y. Matsuoka, T. Iwasaki, N. Nakada, T. Tsuchiyama, S. Takaki, Effect of grain size on thermal and mechanical stability of austenite in metastable austenitic stainless steel, ISIJ Int. 53 (2013) 1224-1230.

[62] J.W. Morris Jr, On the ductile-brittle transition in lath martensitic steel, ISIJ Int. 51 (2011), No. 10, 1569-1575.

[63] H.S. Yang and H.K.D.H. Bhadeshia, Austenite grain size and the martensite-start temperature, Scripta Mater. 60 (2009) 493-495. 
[64] E. Jimenez-Melero, N.H. van Dijk, L. Zhao, J. Sietsma, S.E. Offerman, J.P. Wright, S. van der Zwaag, Martensitic transformation of individual grains in low-alloyed TRIP steels, Scripta Mater. 56 (2007) 421-424.

[65] A.Borgenstam, M.Hillert, J.Ågren, Critical temperature for growth of martensite, Acta metall. mater. 43 (1995) 945-954.

[66] M.Villa, PhD thesis, Isothermal Martensite Formation, Technical University of Denmark, Denmark, 2014. 